\title{
Avermectin toxicity in bovines less than thirty days old
}

\author{
Daniel de Castro Rodrigues ${ }^{\mathrm{a}}$, Carolina Buzullini ${ }^{\mathrm{b}}$, Tiago Arantes Pereira ${ }^{\mathrm{a}}$, Breno Cayeiro Curz ${ }^{\mathrm{b}}$, \\ Lucas Vinicius Costa Gomes ${ }^{\mathrm{b}}$, Vando Edésio Soares ${ }^{\mathrm{c}}$, Thiago Souza Azeredo Bastos ${ }^{\mathrm{d}}$, \\ Luiz Fellipe Monteiro Couto ${ }^{\mathrm{d}}$, Welber Daniel Zanetti Lopes ${ }^{\mathrm{b}, \mathrm{d}, *}$, Gilson Pereira de Oliveira ${ }^{\mathrm{b}}$, \\ Alvimar José da Costa ${ }^{\mathrm{b}}$ \\ ${ }^{a}$ MSD Animal Health, São Paulo, Brazil \\ ${ }^{\mathrm{b}}$ CPPAR - Animal Health Research Center, Faculdade de Ciências Agrárias e Veterinárias, UNESP, Campus de Jaboticabal. Via de acesso Prof. Paulo Donatto Castellane, \\ $s / n^{\circ}$, CEP: 14884-900 Jaboticabal, São Paulo, Brazil \\ ${ }^{\mathrm{c}}$ Universidade Brasil, Campus de Descalvado, CEP 13690-970 São Paulo, SP, Brazil \\ d Instituto de Patologia Tropical e Saúde Pública, Universidade Federal de Goiás (IPTSP/UFG), Goiânia, Goiás 74605050, Brazil
}

\section{A R T I C L E I N F O}

\section{Keywords:}

Abamectin

Calves

Central nervous system

Ivermectin + abamectin

\begin{abstract}
A B S T R A C T
The present study was designed to evaluate possible adverse effects of different dosages of avermectins (abamectin and a combination of ivermectin + abamectin) administered subcutaneously in calves less than one month of age. Clinical as well as biochemical parameters of blood and cerebrospinal fluid (CSF) were evaluated for animals subjected to various treatments. Thirty-five calves were divided into seven groups of five animals each: T01 (control), T02 (abamectin $200 \mu \mathrm{g} / \mathrm{kg}$ ), T03 (abamectin $400 \mu \mathrm{g} / \mathrm{kg}$ ), T04 (abamectin $600 \mu \mathrm{g} / \mathrm{kg}$ ), T05 (ivermectin $450 \mu \mathrm{g} / \mathrm{kg}+$ abamectin $250 \mu \mathrm{g} / \mathrm{kg}$ ), T06 (ivermectin $900 \mu \mathrm{g} / \mathrm{kg}+$ abamectin $500 \mu \mathrm{g} / \mathrm{kg}$ ) and T07 (ivermectin $1350 \mu \mathrm{g} / \mathrm{kg}+$ abamectin $750 \mu \mathrm{g} / \mathrm{kg}$ ). Cerebrospinal fluid and blood samples were collected on day zero (treatment day) and on days 1, 7 and 14 following treatment. The biochemical parameters measured in blood samples were total protein and the AST, GGT and alkaline phosphatase enzymes. In CSF samples, color, aspects, $\mathrm{pH}$, density, cellularity, total proteins, glucose and the CK, ALT and LDH enzymes were evaluated. No neurological toxicity or biochemical changes in the CSF or blood that could be correlated to administration of tested products were observed in this study. Though it is well known that abamectin may lead to intoxication in bovines less than four months of age, based on data from the present study, no adverse clinical effects to the CSF or blood were observed in calves $<30$ days old that had received up to $600 \mu \mathrm{g} / \mathrm{kg}$ abamectin formulations and up to $1350 \mu \mathrm{g} / \mathrm{kg}$ ivermectin $+750 \mu \mathrm{g} / \mathrm{kg}$ abamectin associations. It is possible that the level and activity of the multiple drug resistance gene (MDR1 - $A B C B 1$ ) present in the calves may have influenced the results obtained in this study. Future studies should be carried out.
\end{abstract}

\section{Introduction}

With an increased relevance to parasitology, avermectins and milbemycins appeared in the 1980s. Belonging to the macrocyclic lactone (ML) group, these endectocides act against arthropods and nematodes (Burg et al., 1979).

As part of their mechanism, there is a hypothetical agonist action of gamma amino butyric acid (GABA) that increases the permeability of chlorine ions $\left(\mathrm{Cl}^{-}\right)$, resulting in muscular paralysis (Mellin et al., 1983; Albert et al., 1986). GABA is a known neurotransmitter found in nematodes and arthropods and is also present in the central nervous system (CNS) of mammals. Avermectin concentrations in the CNS of mammals subjected to normal therapeutic dosages presented inexpressible levels of the drug. However, elevated drug levels were verified in the brain tissue of affected bovines and dogs even after application of therapeutic dose (Prichard et al., 2012). Geyer and Janko (2012) describe that the therapeutic safety of macrocyclic lactones in animals is dependent of the expression level and activity of the multidrug efflux transporter MDR1 ( $A B C B 1$ ) gene and P glycoprotein in the blood-brain barrier. Fromm (2004), Marzolini et al. (2004) and Mealey (2004) determined that mutation of the MDR1 (ABCB1) gene alters the pharmacokinetic properties of the P-glycoprotein transported drugs, leading to enhanced bioavailability and reduced drug elimination through the liver, kidney and gut. In othe words, MDR1 mutant dogs,

\footnotetext{
* Corresponding author at: CPPAR - Animal Health Research Center, Faculdade de Ciências Agrárias e Veterinárias, UNESP, Campus de Jaboticabal. Via de acesso Prof. Paulo Donatto Castellane, s/nº, CEP: 14884-900 Jaboticabal, São Paulo, Brazil.

E-mail address: wdzlopes@hotmail.com (W.D.Z. Lopes).
} 
such as Collie, do not express P-glycoprotein at the blood-brain barrier, and for this reason may present severe neurological toxicity after application of therapeutic doses (Geyer et al., 2005)

Despite this understanding reported above, Chrisman (1985), Feldman (1989), Braund (1994) and Wright (1978) describe the hypothesis of analyzing the constituents of the cerebrospinhal fluid (CSF), such the biochemical parameters, in animals that were administered avermectin may reflect physiologic pathological changes in brain functions. This specific research study was motivated by that need.

The present study was designed to evaluate possible adverse effects of varying dosages and formulations, administered subcutaneously, containing different avermectins (abamectin -Duotin ${ }^{\circledR}$ - Merial Animal Health and an association of ivermectin + abamectin- Solution ${ }^{\circledR}$ - MSD Animal Health) in calves $<30$ days old. To accomplish this goal, neurological and clinical parameters, as well the constituents of cerebrospinal fluid (CSF) from animals subjected to these treatments, were analyzed. Secondarily, the biochemical parameter of blood were analyzed in accordance with recommends studies of target animal safety (VICH, 43).

\section{Material and methods}

\subsection{Location, animal selection, animal feeding and allocation of animals into groups}

Prior to implementation, this study was approved by the Ethics Committee for Animal Use (Comissão de Ética no Uso de Animais CEUA) of the School of Agricultural and Veterinarian Sciences (Faculdade de Ciências Agrárias e Veterinárias - FCAV), Jaboticabal campus of the São Paulo State University (Universidade Estadual Paulista "Júlio de Mesquita Filho" - UNESP). It was approved only after being considered in agreement with ethical principles in animal experimentation adopted by the National Council for the Control of Animal Experimentation (Conselho Nacional de Controle de Experimentação Animal - CONCEA).

This experiment was conducted entirely at the Center for Research in Animal Health (Centro de Pesquisas em Sanidade Animal - CPPAR), located on the aforementioned campus.

Thirty-five male Holstein newborn calves were used. The animals were obtained from a commercial farm located in the city of Descalvado, São Paulo State. The calves were separated from their mothers at the moment of birth. At the farm, these animals received colostrum in the first hours of life and were then transported to the CPPAR/FCAV/UNESP where they were kept in pens during the period of the experiment. During the entire experiment, the calves were artificially fed twice per day with four liters of a milk substitute (Amamenta Standard ${ }^{\circledR}$ ) prepared in the proportions recommended by the manufacturer (225 $\mathrm{g}$ of the substitute diluted in two liters of water, heated to a temperature of $39^{\circ} \mathrm{C}$ ). During this period, animals had access to granulated commercial ration (Fri-bezerros ${ }^{\circledast}$ ), coarsely chopped Tifton (Cynodon sp.) hay and ad libitum fresh water. Animals were fed with 70\% Tifton hay and 30\% commercial ration. The amount of food provided was based on $3 \%$ of each animal's body weight. The bromatological composition of this diet was evaluated at the Animal Nutrition Laboratory (Laboratório de Nutrição Animal - FCAV/UNESP).

Each animal was individually weighed, and seven groups were then established with five calves of similar ages and body weights (Table 1). Experimental day zero was defined as the day when the animals were treated. Different formulations containing abamectin (Duotin ${ }^{\circledR}$ - Merial Animal Health) and ivermectin + abamectin (Solution ${ }^{\circledR}$ - MSD Animal Health) were used in this study (Table 1). The animals received the recommended normal dose $(200 \mu \mathrm{g} / \mathrm{kg}$ abamectin; $450 \mu \mathrm{g} / \mathrm{kg}$ ivermectin $+250 \mu \mathrm{g} / \mathrm{kg}$ abamectin), $2 \mathrm{x}(400 \mu \mathrm{g} / \mathrm{kg}$ abamectin; $900 \mu \mathrm{g} / \mathrm{kg}$ ivermectin $+500 \mu \mathrm{g} / \mathrm{kg}$ abamectin) and $3 \mathrm{x}(600 \mu \mathrm{g} / \mathrm{kg}$ abamectin; $1350 \mu \mathrm{g} / \mathrm{kg}$ ivermectin $+750 \mu \mathrm{g} / \mathrm{kg}$ abamectin) as recognized by VICH - 43 (2008). All treatments were administered subcutaneously.
There are no neurologic studies with both formulations, but in accordance with the manufacturer's information's, these products cannot be administered in calves $<16$ weeks ( 4 months) old.

\subsection{Clinical and neurological evaluation}

Identification records were created for each animal, containing the calf's id number, age and body weight. Clinical observations were conducted on day zero (prior to treatment) and on days 1, 7 and 14 post treatment (DPT). On these dates, clinical parameters (cardiac and respiratory frequencies and rectal temperature), eye abnormalities and the cardiovascular, respiratory, muscular-skeletal, integumentary (skin, hair and hooves), gastrointestinal, nervous, lymphatic and urinary systems were examined. During the experiment, all animals were also evaluated for the presence of clinical signs of systemic poisoning, such as ataxia, paresis, dysmetria, ocular changes such as mydriasis, sialorrhea, apathy, prostration, somnolence, excitement, convulsions, dysphonia, diarrhea, dyspnea, jaundice, cough, head tremors, skin tremors and hyperesthesia. If any of these animals had any signs, they would be classified as accented $(+++)$, moderate $(++)$, mild $(+)$ and absent $(-)$.

\subsection{Cerebrospinal fluid evaluations}

\subsubsection{CSF collection}

CSF was obtained through sub-occipital puncture on the cisterna magna, using $20 \mathrm{G}(40 \times 0.9 \mathrm{~mm})$ needles. The liquid was transferred to $1.5 \mathrm{~mL}$ silicone tubes (Eppendorf ${ }^{\circledast}$ ) for analysis of the cytological and biochemical aspects. The animals were immobilized using physical restraint without the use of chemicals (Stober, 1993). Collections were performed before treatments (day zero) and on the 1st, 7th and 14th DPT.

\subsubsection{Physical-chemical evaluation}

Color and aspects of CSF were evaluated in contrast with tubes containing distilled water, both placed against a white background with printed letters. Density was determined using refractometry (refractometer ATAGO Co. LTD., Tokyo, Japan), and indicator strips (Merck do Brasil, Rio de Janeiro, RJ) were used to determine the $\mathrm{pH}$ (Gama, 2003).

\subsubsection{Cytological evaluation}

Global cell counts were conducted in Neubauer chambers, and differential counts for pleocytosis were performed using microscopic preparations obtained through cytocentrifugation (cytocentrifuge FANEM, São Paulo, SP). Afterwards, samples were stained with a methanol May-Grünwald-Giemsa mixture. Global and differential cell counts were performed immediately after obtaining the samples in order to avoid cellular degeneration (Gama, 2003).

\subsubsection{Glycorrhachia analysis}

Glycorrhachia was determined using HK reagent sets (Labtest Diagnóstica, Belo Horizonte, MG) and a biochemical analyzer apparatus (Labquest - Labtest Diagnóstica, Belo Horizonte, MG) by means of colorimetric methods (Gama, 2003).

\subsubsection{Enzymatic analysis}

CSF samples were analyzed for aspartate amino transferase (AST, optimized UV method - IFCC), ${ }^{1}$ creatine kinase (CK, kinetic UV method - IFCC) ${ }^{2}$ and total protein (using the total protein reagents ${ }^{1}$ ). Readings were performed in an automatic spectrophotometer ${ }^{2}$.

Determinations of CK, AST and lactate dehydrogenase activities

\footnotetext{
${ }^{1}$ Wiener Lab. - Rosario, Argentina.

${ }^{2}$ Automatic biochemical analyzer, Cobas Mira S, Roche do Brasil - São Paulo, SP.
} 
Table 1

Experimental design.

\begin{tabular}{|c|c|c|c|c|}
\hline Group & $\mathrm{N}^{\circ}$ of bovines & Mean age (days) & Treatment & Dose \\
\hline I & 5 & 22.1 & Saline solution & saline $0,9 \%$ \\
\hline II & 5 & 22.3 & Abamectin & $200 \mu \mathrm{g} / \mathrm{kg}$ (recommended dose) \\
\hline III & 5 & 22.0 & Abamectin & $400 \mu \mathrm{g} / \mathrm{kg}$ \\
\hline IV & 5 & 22.4 & Abamectin & $600 \mu \mathrm{g} / \mathrm{kg}$ \\
\hline $\mathrm{V}$ & 5 & 22.3 & Ivermectin $2.25 \%+$ Abamectin $1.25 \%$ & $450 \mu \mathrm{g} / \mathrm{kg}$-ivermectin $250 \mu \mathrm{g} / \mathrm{kg}$-abamectin (recommended dose) \\
\hline VI & 5 & 22.6 & Ivermectin $2.25 \%+$ Abamectin $1.25 \%$ & $900 \mu \mathrm{g} / \mathrm{kg}$-ivermectin $500 \mu \mathrm{g} / \mathrm{kg}$-abamectin \\
\hline VII & 5 & 22.2 & Ivermectin $2.25 \%+$ Abamectin $1.25 \%$ & $1350 \mu \mathrm{g} / \mathrm{kg}$-ivermectin $750 \mu \mathrm{g} / \mathrm{kg}$-abamectin \\
\hline
\end{tabular}

Table 2

Variation of the mean values of the clinical parameters of bovine animals under 30 days submitted to different concentrations of avermectin.

\begin{tabular}{|c|c|c|c|c|c|c|c|c|c|}
\hline \multirow[t]{2}{*}{ Group } & \multirow[t]{2}{*}{ Treatment } & \multicolumn{8}{|c|}{ Experimental day/Rectal temperature ${ }^{\circ} \mathrm{C}$} \\
\hline & & ZERO & & 1 & & 7 & & 14 & \\
\hline I & Saline solution & 38.26 & B & 38.76 & A & 38.60 & A & 39.06 & A \\
\hline II & Abamectin $(200 \mu \mathrm{g})$ - recommended dose & 39.38 & $\mathrm{AB}$ & 38.38 & A & 39.20 & A & 38.46 & A \\
\hline III & Abamectin $(400 \mu \mathrm{g})$ & 38.40 & B & 38.66 & A & 38.34 & A & 38.78 & A \\
\hline IV & Abamectin $(600 \mu g)$ & 38.18 & B & 38.62 & A & 38.50 & A & 38.82 & A \\
\hline $\mathrm{V}$ & Ivermectin $(450 \mu \mathrm{g})+$ Abamectin $(250 \mu \mathrm{g})-$ recommended dose & 39.66 & A & 39.24 & A & 38.96 & A & 39.06 & A \\
\hline VI & Ivermectin $(900 \mu g)+$ Abamectin $(500 \mu g)$ & 38.18 & B & 38.70 & A & 38.32 & A & 39.28 & A \\
\hline VII & Ivermectin $(1350 \mu \mathrm{g})+$ Abamectin $(750 \mu \mathrm{g})$ & 38.64 & $\mathrm{AB}$ & 38.76 & A & 38.44 & A & 39.68 & A \\
\hline \multirow[t]{2}{*}{ Group } & Treatment & \multicolumn{8}{|c|}{ Experimental day/Heart frequency (BPM) } \\
\hline & & ZERO & & 1 & & 7 & & 14 & \\
\hline I & Saline solution & 89.60 & A & 82.40 & A & 77.60 & $\mathrm{AB}$ & 85.60 & A \\
\hline II & Abamectin $(200 \mu \mathrm{g})$ - recommended dose & 68.80 & B & 80.00 & A & 68.80 & B & 77.60 & A \\
\hline III & Abamectin $(400 \mu \mathrm{g})$ & 77.60 & $\mathrm{AB}$ & 82.40 & A & 83.20 & $\mathrm{AB}$ & 85.60 & A \\
\hline IV & Abamectin $(600 \mu g)$ & 75.20 & B & 82.40 & A & 83.20 & $\mathrm{AB}$ & 84.80 & A \\
\hline $\mathrm{V}$ & Ivermectin $(450 \mu \mathrm{g})+$ Abamectin $(250 \mu \mathrm{g})-$ recommended dose & 67.20 & $\mathrm{~B}$ & 83.20 & A & 70.00 & $\mathrm{AB}$ & 70.40 & A \\
\hline VI & Ivermectin $(900 \mu g)+$ Abamectin $(500 \mu g)$ & 63.20 & B & 85.60 & A & 81.60 & $\mathrm{AB}$ & 85.60 & A \\
\hline VII & Ivermectin $(1350 \mu \mathrm{g})+$ Abamectin $(750 \mu \mathrm{g})$ & 89.60 & A & 80.80 & A & 84.80 & A & 84.00 & A \\
\hline \multirow[t]{2}{*}{ Group } & Treatment & \multicolumn{8}{|c|}{ Experimental day/Respiratory frequency (MPM) } \\
\hline & & ZERO & & 1 & & 7 & & 14 & \\
\hline I & Saline solution & 20.80 & $\mathrm{AB}$ & 23.20 & A & 23.20 & A & 25.60 & A \\
\hline II & Abamectin $(200 \mu \mathrm{g})$ - recommended dosel & 21.60 & $\mathrm{AB}$ & 25.60 & A & 20.80 & A & 27.20 & A \\
\hline III & Abamectin $(400 \mu \mathrm{g})$ & 20.00 & $\mathrm{AB}$ & 22.40 & A & 24.00 & A & 25.60 & A \\
\hline IV & Abamectin $(600 \mu g)$ & 21.60 & $\mathrm{AB}$ & 20.00 & A & 20.80 & A & 24.00 & A \\
\hline $\mathrm{V}$ & Ivermectin $(450 \mu \mathrm{g})+$ Abamectin $(250 \mu \mathrm{g})-$ recommended dose & 24.80 & A & 24.00 & A & 22.40 & A & 25.60 & A \\
\hline VI & Ivermectin $(900 \mu \mathrm{g})+$ Abamectin $(500 \mu \mathrm{g})$ & 18.40 & B & 23.20 & A & 23.20 & A & 25.60 & A \\
\hline VII & Ivermectin $(1350 \mu \mathrm{g})+$ Abamectin $(750 \mu \mathrm{g})$ & 22.40 & $\mathrm{AB}$ & 22.40 & A & 22.40 & A & 24.00 & A \\
\hline
\end{tabular}

Means values followed by the same letter on the same colun do not differ significantly at a $95 \%$ reliability level.

were performed using a reagent set (Labtest) and the kinetic method, with readings obtained using a biochemical analyzer apparatus (Labquest - Labtest Diagnóstica, Belo Horizonte, MG) (Gama, 2003).

\subsubsection{Protein analysis}

Determination of total protein values was performed using Sensi Prot reagent sets (Labtest Diagnóstica, Belo Horizonte, MG) and the pyrogallol red method, with readings obtained using a biochemical analyzer apparatus (Labquest - Labtest Diagnóstica, Belo Horizonte, MG) (Gama, 2003).

\subsection{Blood enzyme and protein evaluations}

To separate the blood serum for biochemical examination, $10 \mathrm{~mL}$ of blood was collected in vials without additives. This procedure was performed based on the recommendations of the Target Animal for Veterinary Pharmaceutical Products (VICH 43 -, 2008). The samples were analyzed for alkaline phosphatase (AP, Bowers \& McComb modified method), ${ }^{3}$ aspartate amino transferase (AST, optimized UV method - IFCC) ${ }^{4}$ and gamma glutamyl transferase (GGT kinetic

\footnotetext{
${ }^{3}$ Labtest Diagnóstica S.A. - Lagoa Santa, MG

${ }^{4}$ Wiener Lab. - Rosario, Argentina.
}

method $)^{1}$. Readings were obtained using an automatic spectrophotometer. Determination of total protein values was conducted using total protein reagents ${ }^{1}$.

\subsection{Data analysis}

A design with parcels subdivided in time ("Split Plot in Time") was adopted, considering the treated and control groups, each with five repetitions, as the main treatments and observation dates as the secondary treatments.

Data were analyzed using a methodology proposed by Little and Hills (1978), which required logarithmic transformation $(\log [x+1])$. Analyses were performed by applying the F-Test, and averages were compared using the Tukey test (SAS, 1996).

\section{Results and discussion}

\subsection{Clinical examinations}

Clinical symptoms due to different treatment formulations or dosages were not observed in any of the experimental animals.

Cases of lactone intoxication in bovine animals are expected to demonstrate neurological toxicity in animals; however, in the present 

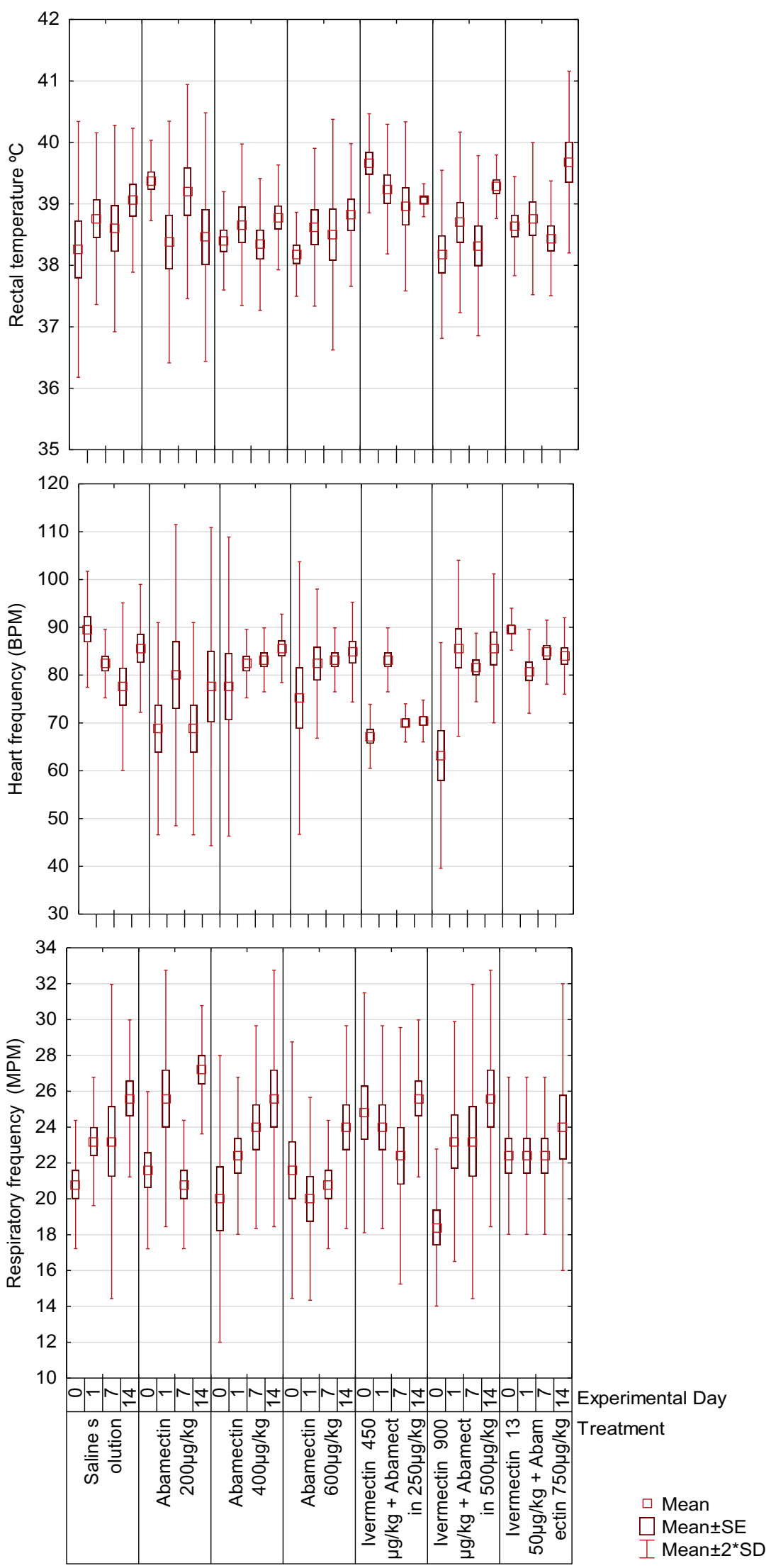

Fig. 1. Boxplot of clinical parameters of bovine animals under 30 days submitted to different concentrations of avermectin. 
Table 3

Data analysis of the cerebrospinal fluid parametres of bovine animals under 30 days submitted to different concentrations of avermectin.

\begin{tabular}{|c|c|c|c|c|c|c|c|c|c|}
\hline \multirow[t]{2}{*}{ Group } & \multirow[t]{2}{*}{ Treatment } & \multicolumn{8}{|c|}{ Experimental day/Density value (Cerebrospinal fluid) } \\
\hline & & ZERO & & 1 & & 7 & & 14 & \\
\hline I & Saline solution & 1.005 & A & 1.005 & A & 1.005 & A & 1.005 & A \\
\hline II & Abamectin $(200 \mu \mathrm{g})$ - recommended dose & 1.005 & A & 1.005 & A & 1.006 & A & 1.005 & A \\
\hline III & Abamectin $(400 \mu \mathrm{g})$ & 1.006 & A & 1.005 & A & 1.005 & A & 1.005 & A \\
\hline IV & Abamectin $(600 \mu \mathrm{g})$ & 1.006 & A & 1.005 & A & 1.005 & A & 1.005 & A \\
\hline V & Ivermectin $(450 \mu \mathrm{g})+$ Abamectin $(250 \mu \mathrm{g})-$ recommended dose & 1.006 & A & 1.007 & A & 1.006 & A & 1.005 & A \\
\hline VI & Ivermectin $(900 \mu \mathrm{g})+$ Abamectin $(500 \mu \mathrm{g})$ & 1.005 & A & 1.005 & A & 1.005 & A & 1.005 & A \\
\hline VII & Ivermectin $(1350 \mu \mathrm{g})+$ Abamectin $(750 \mu \mathrm{g})$ & 1.005 & A & 1.005 & A & 1.005 & A & 1.005 & A \\
\hline \multirow[t]{2}{*}{ Group } & Treatment & \multicolumn{8}{|c|}{ Experimental day/pH (Cerebrospinal fluid) } \\
\hline & & ZERO & & 1 & & 7 & & 14 & \\
\hline I & Saline solution & 7.10 & $\mathrm{AB}$ & 8.00 & A & 7.90 & A & 8.00 & A \\
\hline II & Abamectin $(200 \mu g)$ - recommended dosel & 7.80 & A & 7.70 & A & 7.80 & A & 8.00 & A \\
\hline III & Abamectin $(400 \mu g)$ & 7.40 & $\mathrm{AB}$ & 8.00 & A & 7.70 & A & 8.00 & A \\
\hline IV & Abamectin $(600 \mu g)$ & 7.00 & B & 7.80 & A & 8.00 & A & 7.80 & A \\
\hline V & Ivermectin $(450 \mu \mathrm{g})+$ Abamectin $(250 \mu \mathrm{g})-$ recommended dose & 7.40 & $\mathrm{AB}$ & 6.80 & B & 7.80 & A & 7.60 & A \\
\hline VI & Ivermectin $(900 \mu \mathrm{g})+$ Abamectin $(500 \mu \mathrm{g})$ & 7.20 & $\mathrm{AB}$ & 7.90 & A & 7.70 & A & 7.80 & A \\
\hline VII & Ivermectin $(1350 \mu \mathrm{g})+$ Abamectin $(750 \mu \mathrm{g})$ & 7.30 & $\mathrm{AB}$ & 8.00 & A & 8.00 & A & 8.00 & A \\
\hline \multirow[t]{2}{*}{ Group } & Treatment & \multicolumn{8}{|c|}{ Experimental day/Glucose (Cerebrospinal fluid) } \\
\hline & & ZERO & & 1 & & 7 & & 14 & \\
\hline I & Saline solution & 33.20 & A & 56.20 & A & 52.40 & A & 46.40 & A \\
\hline II & Abamectin $(200 \mu \mathrm{g})$ - recommended dose & 54.20 & A & 43.40 & A & 56.80 & A & 41.14 & A \\
\hline III & Abamectin $(400 \mu \mathrm{g})$ & 55.60 & A & 59.60 & A & 57.20 & A & 56.80 & A \\
\hline IV & Abamectin $(600 \mu g)$ & 45.00 & A & 54.20 & A & 47.00 & A & 46.40 & A \\
\hline $\mathrm{V}$ & Ivermectin $(450 \mu \mathrm{g})+$ Abamectin $(250 \mu \mathrm{g})-$ recommended dose & 57.20 & A & 43.40 & A & 51.00 & A & 37.20 & A \\
\hline VI & Ivermectin $(900 \mu g)+$ Abamectin $(500 \mu g)$ & 46.80 & A & 48.80 & A & 49.20 & A & 45.00 & A \\
\hline VII & Ivermectin $(1350 \mu \mathrm{g})+$ Abamectin $(750 \mu \mathrm{g})$ & 48.60 & A & 51.40 & A & 50.80 & A & 43.20 & A \\
\hline \multirow[t]{2}{*}{ Group } & Treatment & \multicolumn{8}{|c|}{ Experimental day/Leukocytes (Cerebrospinal fluid) } \\
\hline & & ZERO & & 1 & & 7 & & 14 & \\
\hline I & Saline solution & 2.00 & A & 2.00 & B & 2.90 & A & 2.40 & A \\
\hline II & Abamectin $(200 \mu \mathrm{g})$ - recommended dose & 0.80 & A & 5.00 & B & 2.80 & A & 2.40 & A \\
\hline III & Abamectin $(400 \mu \mathrm{g})$ & 0.50 & A & 0.50 & $\mathrm{~B}$ & 0.00 & A & 1.00 & A \\
\hline IV & Abamectin $(600 \mu g)$ & 0.50 & A & 0.00 & B & 1.00 & A & 0.50 & A \\
\hline $\mathrm{V}$ & Ivermectin $(450 \mu \mathrm{g})+$ Abamectin $(250 \mu \mathrm{g})-$ recommended dose & 4.40 & A & 17.50 & A & 1.00 & A & 7.80 & A \\
\hline VI & Ivermectin $(900 \mu \mathrm{g})+$ Abamectin $(500 \mu \mathrm{g})$ & 2.00 & A & 2.50 & $\mathrm{~B}$ & 2.00 & A & 1.00 & A \\
\hline VII & Ivermectin $(1350 \mu \mathrm{g})+$ Abamectin $(750 \mu \mathrm{g})$ & 0.50 & A & 0.50 & $\mathrm{~B}$ & 0.00 & A & 0.60 & A \\
\hline \multirow[t]{2}{*}{ Group } & Treatment & \multicolumn{8}{|c|}{ Experimental day/LDH (Cerebrospinal fluid) } \\
\hline & & ZERO & & 1 & & 7 & & 14 & \\
\hline I & Saline solution & 0.6417 & A & 1.1572 & A & 0.2461 & A & 0.5498 & A \\
\hline II & Abamectin $(200 \mu \mathrm{g})$ - recommended dose & 0.8535 & A & 1.0043 & $\mathrm{~A}$ & 1.2721 & A & 1.4033 & A \\
\hline III & Abamectin $(400 \mu \mathrm{g})$ & 1.0219 & A & 0.8167 & A & 0.5333 & A & 0.8527 & A \\
\hline IV & Abamectin $(600 \mu \mathrm{g})$ & 0.4922 & A & 1.0172 & A & 0.2644 & A & 0.8596 & A \\
\hline $\mathrm{V}$ & Ivermectin $(450 \mu \mathrm{g})+$ Abamectin $(250 \mu \mathrm{g})-$ recommended dose & 1.0763 & A & 1.2365 & A & 1.5898 & A & 0.6663 & A \\
\hline VI & Ivermectin $(900 \mu \mathrm{g})+$ Abamectin $(500 \mu \mathrm{g})$ & 0.2461 & A & 1.0748 & A & 0.6113 & A & 0.8189 & A \\
\hline VII & Ivermectin $(1350 \mu \mathrm{g})+$ Abamectin $(750 \mu \mathrm{g})$ & 0.9844 & A & 0.6391 & A & 0.2910 & A & 0.6379 & A \\
\hline Group & Treatment & Experim & day/ & Cerebrosp & uid) & & & & \\
\hline & & ZERO & & 1 & & 7 & & 14 & \\
\hline I & Saline solution & 23.05 & A & 34.92 & A & 34.06 & $\mathrm{AB}$ & 9.42 & A \\
\hline II & Abamectin $(200 \mu \mathrm{g})$ - recommended dose & 17.51 & A & 26.19 & A & 40.94 & A & 17.81 & A \\
\hline III & Abamectin $(400 \mu \mathrm{g})$ & 24.15 & A & 29.14 & A & 14.27 & B & 16.37 & A \\
\hline IV & Abamectin $(600 \mu g)$ & 23.36 & A & 24.55 & A & 11.78 & B & 19.82 & A \\
\hline $\mathrm{V}$ & Ivermectin $(450 \mu \mathrm{g})+$ Abamectin $(250 \mu \mathrm{g})-$ recommended dose & 18.41 & A & 37.55 & A & 54.90 & A & 27.44 & A \\
\hline VI & Ivermectin $(900 \mu g)+$ Abamectin $(500 \mu g)$ & 26.76 & A & 37.13 & A & 14.92 & B & 17.38 & A \\
\hline VII & Ivermectin $(1350 \mu \mathrm{g})+$ Abamectin $(750 \mu \mathrm{g})$ & 21.77 & A & 29.15 & A & 13.43 & B & 14.83 & A \\
\hline Group & Treatment & Experim & day/ & rebrospin & & & & & \\
\hline & & ZERO & & 1 & & 7 & & 14 & \\
\hline I & Saline solution & 43.65 & A & 33.94 & A & 52.91 & A & 29.12 & A \\
\hline II & Abamectin $(200 \mu \mathrm{g})$ - recommended dose & 43.37 & A & 38.86 & A & 43.20 & A & 39.86 & A \\
\hline III & Abamectin $(400 \mu \mathrm{g})$ & 58.28 & A & 29.09 & A & 53.03 & A & 33.96 & A \\
\hline IV & Abamectin $(600 \mu g)$ & 48.57 & A & 24.23 & A & 48.23 & A & 38.76 & A \\
\hline $\mathrm{V}$ & Ivermectin $(450 \mu \mathrm{g})+$ Abamectin $(250 \mu \mathrm{g})-$ recommended dose & 53.14 & A & 43.54 & A & 38.40 & A & 29.15 & A \\
\hline VI & Ivermectin $(900 \mu \mathrm{g})+$ Abamectin $(500 \mu \mathrm{g})$ & 38.84 & A & 43.60 & $\mathrm{~A}$ & 33.83 & A & 38.94 & A \\
\hline VII & Ivermectin $(1350 \mu \mathrm{g})+$ Abamectin $(750 \mu \mathrm{g})$ & 43.70 & A & 53.26 & A & 46.80 & A & 33.95 & A \\
\hline Group & Treatment & Experim & day/ & protein $(C$ & sspir & iid) & & & \\
\hline & & ZERO & & 1 & & 7 & & 14 & \\
\hline I & Saline solution & 32.46 & A & 26.08 & A & 23.14 & A & 23.18 & A \\
\hline II & Abamectin $(200 \mu \mathrm{g})$ - recommended dose & 22.38 & A & 9.10 & A & 27.48 & A & 20.22 & A \\
\hline
\end{tabular}


Table 3 (continued)

\begin{tabular}{|c|c|c|c|c|c|c|c|c|c|}
\hline \multirow[t]{2}{*}{ Group } & \multirow[t]{2}{*}{ Treatment } & \multicolumn{8}{|c|}{ Experimental day/Density value (Cerebrospinal fluid) } \\
\hline & & ZERO & & 1 & & 7 & & 14 & \\
\hline III & Abamectin $(400 \mu g)$ & 18.20 & A & 18.86 & A & 19.60 & A & 28.04 & A \\
\hline IV & Abamectin $(600 \mu \mathrm{g})$ & 17.42 & A & 19.44 & A & 18.44 & A & 26.68 & A \\
\hline $\mathrm{V}$ & Ivermectin $(450 \mu \mathrm{g})+$ Abamectin $(250 \mu \mathrm{g})-$ recommended dose & 41.24 & A & 28.95 & A & 19.66 & A & 22.52 & A \\
\hline VI & Ivermectin $(900 \mu \mathrm{g})+$ Abamectin $(500 \mu \mathrm{g})$ & 21.94 & A & 47.50 & A & 27.86 & A & 24.88 & A \\
\hline VII & Ivermectin $(1350 \mu \mathrm{g})+$ Abamectin $(750 \mu \mathrm{g})$ & 17.40 & A & 23.40 & A & 25.72 & A & 17.30 & A \\
\hline
\end{tabular}

Means values followed by the same letter on the same colun do not differ significantly at a $95 \%$ reliability level.
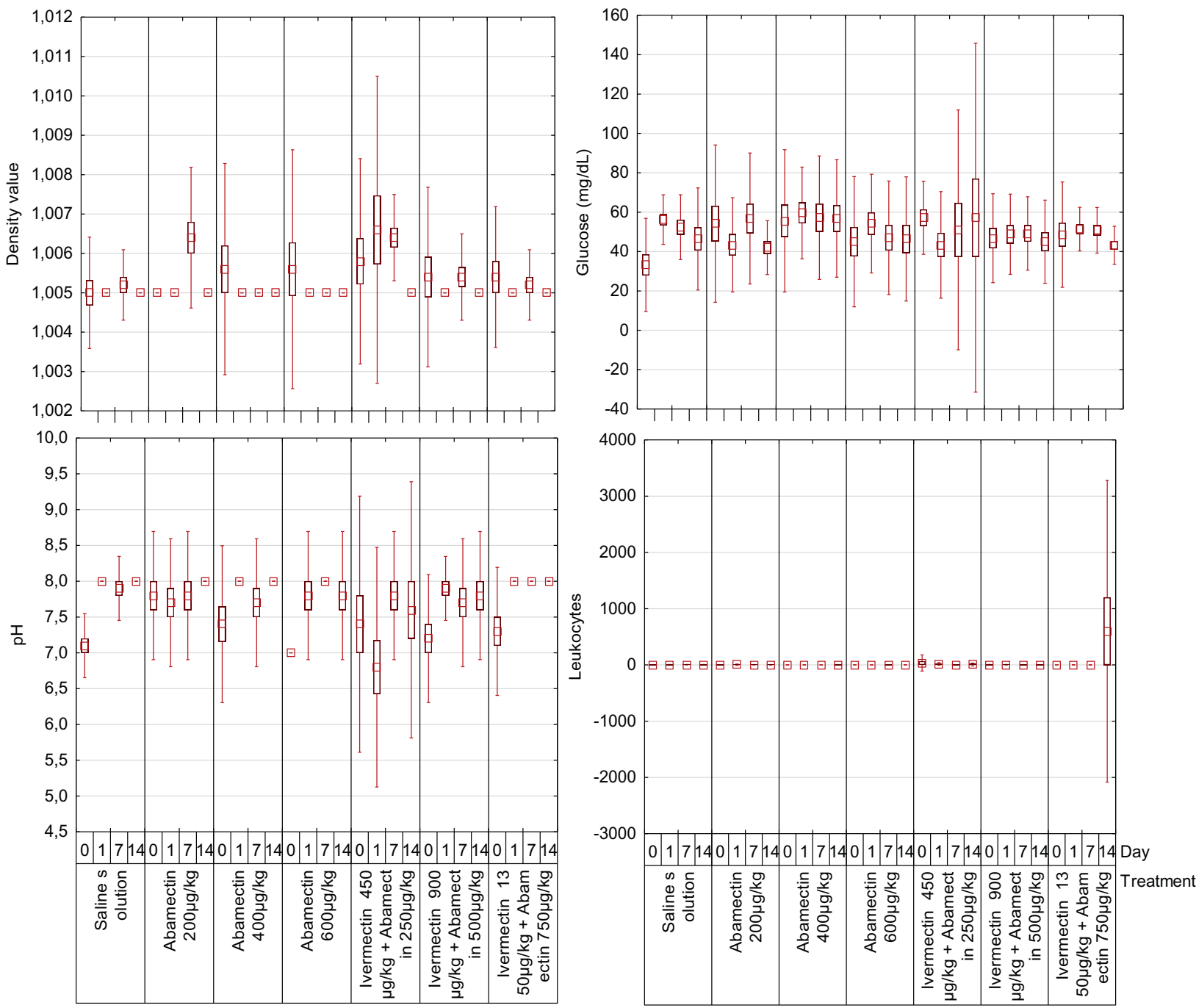

$\square$ Mean

I Mean $\pm 2 *$ SD

Fig. 2. (a) and (b). Boxplot of the cerebrospinal fluid parametres of bovine animals under 30 days submitted to different concentrations of avermectin.

study, no neurological abnormalities were observed in calves. In experiments which induced acute and chronic abamectin intoxication in mice, rabbits, dogs and monkeys, animals presented mainly mydriasis, vomiting, tremors, ataxia, depression, seizures, coma and death (Lankas and Gordon, 1989). Evaluating tolerance of calves to different doses of abamectin ( 0.3 to $8 \mathrm{mg} / \mathrm{kg}$ ), Button et al. (1988) mostly observed signs of depression and ataxia. In this case, moderate intoxication symptoms were detected in animals after receiving dosages starting at $0.727 \mathrm{mg}$ / $\mathrm{kg}$. When the same researchers administered volumes between 2 and $8 \mathrm{mg} / \mathrm{kg}$, toxicity signs such as ataxia, progressive paresis, mydriasis, decubitus, sialorrhea and death occurred. On the present study, evaluating clinical safety of different formulations containing abamectin (Duotin ${ }^{\circledR}$, MerialSaúde Animal,and Solution ${ }^{\circledR}$, MSD Saúde Animal), it was possible to observe that the highest dosages for each formulation containing this active component were $0.6 \mathrm{mg} / \mathrm{kg}$ and $0.75 \mathrm{mg} / \mathrm{kg}$, respectively. Independent of administered dosages, it was not possible to verify any signs of neurologic toxicity in animals.

A possible explanation for calves on the present study not demonstrating signs of neurologic toxicity by different abamectin dosages may be found in more advanced studies with the MDR1 gene and P-glycoprotein, conducted in dogs. Dogs which showed toxicity signs to some macrocyclic lactones administered in therapeutic dosages may present a mutation on the MDR1 gene and become more sensible to such active principles. This gene (MDR1) codifies P-glycoprotein, which in turn functions as a cellular efflux pump for various medications in several species of animals (Geyer and Janko, 2012). 


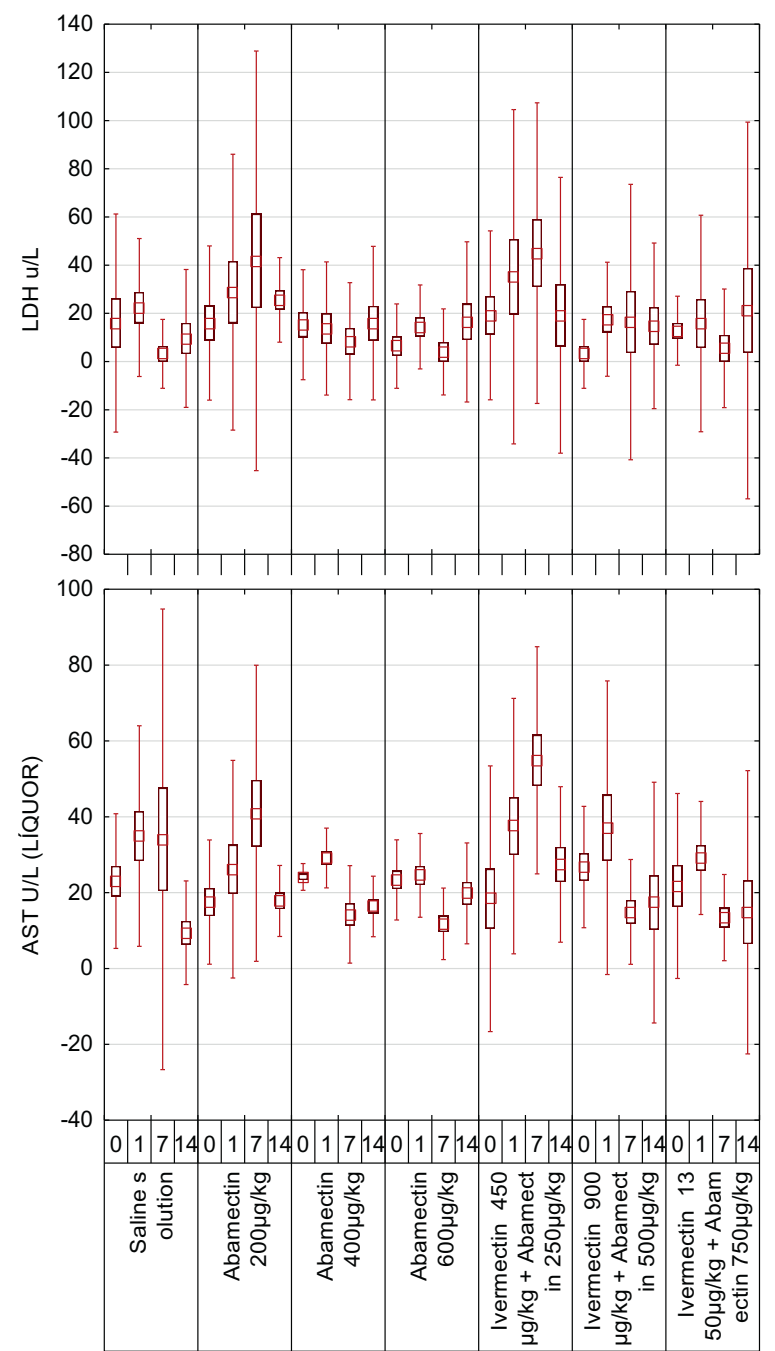

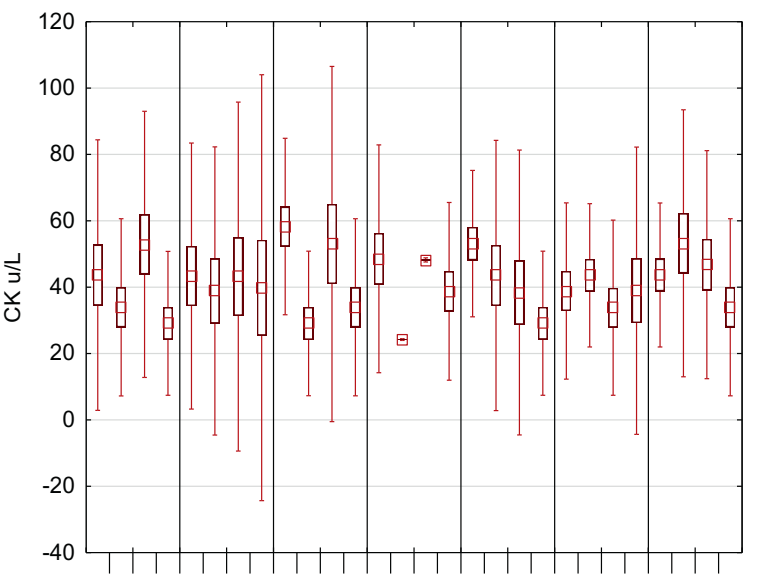

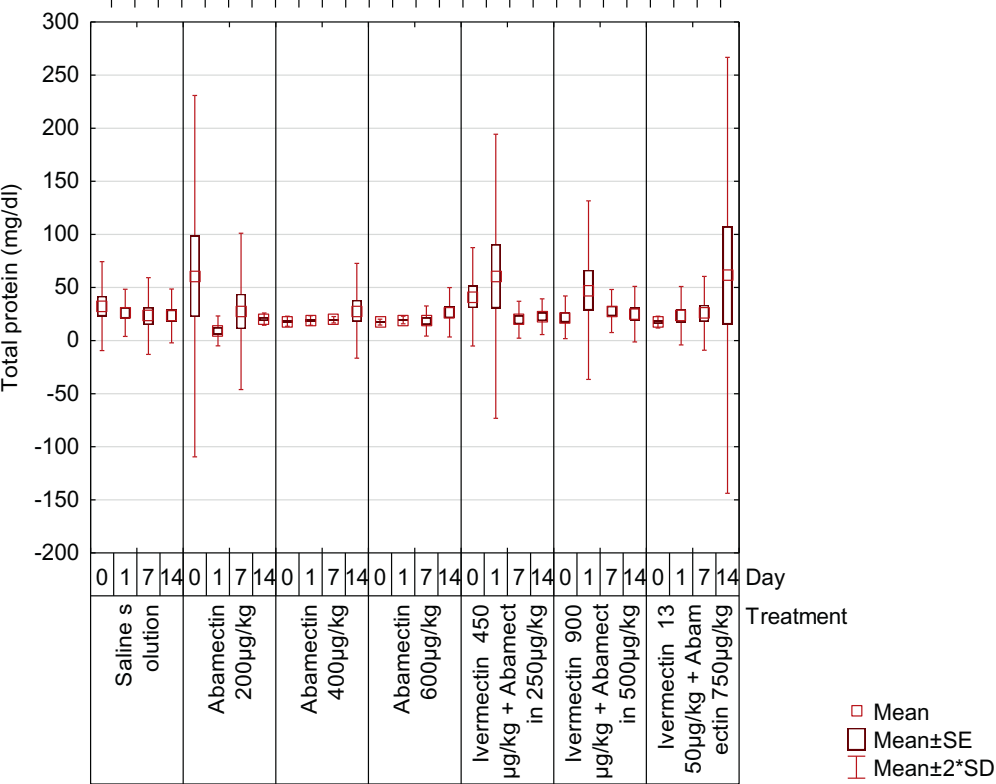

Fig. 2. (continued)
According to Fecht et al. (2007), the gene is located on chromosome 14 and is composed by 28 exons. Mutation consists in deletion of the fourth exon, which results in synthesis of defective and nonfunctional Pglycoprotein, making the bearer of this mutation susceptible to intoxication, even when receiving an active component in its therapeutic dosages (Bissonnette et al., 2008). The animal may be homozygous (MDR1 + $/+$ ) for the MDR1 gene, and in this case it is considered normal (or wild) and do not present the same risk as homozygous mutants. Homozygous mutant animals (MDR1 -/-) present extreme sensibility and predisposition to intoxication. Heterozygous individuals (MDR1 $+/-$ ) are bearing the mutation, being either susceptible to intoxication or not (Bissonnette et al., 2008).

It is known from animal models (Schinkel et al., 1997) that MDR1 is up-regulated in the first weeks of life. Therefore, a hypothesis that could justify the results found in the present study is that if the MDR1 expression level in young calves is already fully regulated, there is sufficient protection against brain penetration by ML in these animals. However, further studies should be conducted to confirm this hypothesis.

Based on results expressed in Table 2 and Fig. 1, it is possible to verify that at no time during this study were significant differences observed for respiratory frequencies and rectal temperatures in experimental calves; these clinical parameters remained within normal ranges as preconized by Feitosa (2008). Concerning the average values of cardiac frequencies measured in these animals, significant differences ( $p \leq 0.05$ ) were observed between groups on days zero (before treatment) and 7 (7th DPT). Groups of calves with mean cardiac frequency values above normal levels for this species were observed on all days post-treatment. However, this condition also occurred occasionally in animals from the untreated control group (T01 - saline solution, Table 2 ad Fig. 1). Another aspect that must be considered is the stress of animals submitted to physical restraint for sample collection. In addition, rectal temperatures and other physiological variables, such as age, may alter the vagal state, intensifying activity of the cardio-accelerator and vasoconstrictor centers and therefore elevating cardiac rates.

Seixas et al. (2006) reported the occurrence of several intoxication outbreaks caused by abamectin, totaling at least 57 deaths over four years (including adult animals), reported from just three diagnostic services contacted in Brazil. This research study emphasized the possibility that avermectin intoxications are frequent and that the numbers of iatrogenic intoxications are probably being underestimated.

On the other hand, it is essential to reinforce that these cases reported by Seixas et al. (2006) were diagnosed based on administration records and observation of clinical symptoms after treatment. In most cases, exact information regarding animal ages, administered dosages and clinical characteristics were not available. Those researchers highlighted the importance of performing in-depth biochemical and neurological studies in order to better understand possible adverse effects of abamectin in bovines.

Benz and Cox (1989) and Courtney and Roberson (1995) considered the safety margin of avermectins to be elevated, even in animals highly 
Table 4

Data analysis of the biochemical parameters performed to bovine animals under 30 days submitted to different concentrations of avermectin.

\begin{tabular}{|c|c|c|c|c|c|c|c|c|c|}
\hline \multirow[t]{2}{*}{ Group } & \multirow[t]{2}{*}{ Treatment } & \multicolumn{8}{|c|}{ Experimental day/Total serum protein $(\mathrm{g} / \mathrm{dL})$} \\
\hline & & ZERO & & 1 & & 7 & & 14 & \\
\hline I & Saline solution & 6.86 & A & 6.99 & A & 6.88 & A & 7.09 & A \\
\hline II & Abamectin $(200 \mu \mathrm{g})$ - recommended dosel & 7.41 & A & 7.15 & A & 7.30 & A & 7.22 & A \\
\hline III & Abamectin $(400 \mu \mathrm{g})$ & 6.52 & $\mathrm{~A}$ & 6.88 & A & 6.73 & A & 6.72 & A \\
\hline IV & Abamectin $(600 \mu \mathrm{g})$ & 6.96 & A & 7.04 & A & 6.90 & A & 7.16 & A \\
\hline $\mathrm{V}$ & Ivermectin $(450 \mu \mathrm{g})+$ Abamectin $(250 \mu \mathrm{g})-$ recommended dose & 7.39 & A & 7.01 & A & 7.14 & A & 6.32 & A \\
\hline VI & Ivermectin $(900 \mu \mathrm{g})+$ Abamectin $(500 \mu \mathrm{g})$ & 7.18 & A & 7.11 & A & 6.80 & A & 7.18 & A \\
\hline VII & Ivermectin $(1350 \mu \mathrm{g})+$ Abamectin $(750 \mu \mathrm{g})$ & 7.39 & A & 7.74 & A & 7.47 & A & 7.58 & A \\
\hline \multirow[t]{2}{*}{ Group } & Treatment & \multicolumn{8}{|c|}{ Experimental day/AST (g/dL) } \\
\hline & & ZERO & & 1 & & 7 & & 14 & \\
\hline I & Saline solution & 55.57 & A & 52.83 & A & 55.47 & A & 81.16 & A \\
\hline II & Abamectin $(200 \mu \mathrm{g})$ - recommended dose & 58.67 & A & 86.81 & A & 71.23 & A & 63.87 & A \\
\hline III & Abamectin $(400 \mu \mathrm{g})$ & 54.94 & A & 49.25 & A & 52.40 & A & 44.71 & A \\
\hline IV & Abamectin $(600 \mu \mathrm{g})$ & 49.70 & A & 53.39 & A & 55.48 & A & 55.04 & A \\
\hline $\mathrm{V}$ & Ivermectin $(450 \mu \mathrm{g})+$ Abamectin $(250 \mu \mathrm{g})-$ recommended dose & 88.95 & A & 143.69 & A & 44.00 & A & 53.89 & A \\
\hline VI & Ivermectin $(900 \mu \mathrm{g})+$ Abamectin $(500 \mu \mathrm{g})$ & 59.83 & A & 48.15 & A & 54.50 & A & 61.21 & A \\
\hline VII & Ivermectin $(1350 \mu \mathrm{g})+$ Abamectin $(750 \mu \mathrm{g})$ & 55.39 & A & 48.89 & A & 65.72 & A & 52.94 & A \\
\hline \multirow[t]{2}{*}{ Group } & Treatment & \multicolumn{8}{|c|}{ Experimental day/GGT (g/dL) } \\
\hline & & ZERO & & 1 & & 7 & & 14 & \\
\hline I & Saline solution & 36.40 & A & 36.40 & A & 28.80 & A & 33.80 & A \\
\hline II & Abamectin $(200 \mu \mathrm{g})$ - recommended dose & 32.60 & A & 42.60 & A & 57.00 & A & 54.40 & A \\
\hline III & Abamectin $(400 \mu \mathrm{g})$ & 47.80 & A & 36.40 & A & 28.00 & A & 29.80 & A \\
\hline IV & Abamectin $(600 \mu \mathrm{g})$ & 39.00 & A & 37.78 & A & 32.60 & A & 32.40 & A \\
\hline V & Ivermectin $(450 \mu \mathrm{g})+$ Abamectin $(250 \mu \mathrm{g})-$ recommended dose of label & 58.00 & A & 60.40 & A & 41.40 & A & 40.20 & A \\
\hline VI & Ivermectin $(900 \mu \mathrm{g})+$ Abamectin $(500 \mu \mathrm{g})$ & 25.80 & A & 56.80 & A & 41.60 & A & 41.40 & A \\
\hline VII & Ivermectin $(1350 \mu \mathrm{g})+$ Abamectin $(750 \mu \mathrm{g})$ & 29.80 & A & 32.40 & A & 30.00 & A & 38.80 & A \\
\hline \multirow[t]{2}{*}{ Group } & Treatment & \multicolumn{8}{|c|}{ Experimental day/FA (g/dL) } \\
\hline & & ZERO & & 1 & & 7 & & 14 & \\
\hline I & Saline solution & 131.25 & $\mathrm{BC}$ & 96.01 & $\mathrm{~B}$ & 107.20 & A & 109.45 & A \\
\hline II & Abamectin $(200 \mu \mathrm{g})$ - recommended dose & 104.64 & C & 134.32 & $\mathrm{AB}$ & 137.66 & A & 131.02 & A \\
\hline III & Abamectin $(400 \mu \mathrm{g})$ & 163.58 & $\mathrm{ABC}$ & 184.36 & A & 178.80 & A & 186.46 & A \\
\hline IV & Abamectin $(600 \mu \mathrm{g})$ & 226.70 & A & 195.22 & A & 164.12 & A & 172.16 & A \\
\hline $\mathrm{V}$ & Ivermectin $(450 \mu \mathrm{g})+$ Abamectin $(250 \mu \mathrm{g})-$ recommended dose & 118.42 & $\mathrm{C}$ & 169.16 & $\mathrm{AB}$ & 145.92 & A & 145.96 & A \\
\hline VI & Ivermectin $(900 \mu \mathrm{g})+$ Abamectin $(500 \mu \mathrm{g})$ & 201.80 & $\mathrm{AB}$ & 154.74 & $\mathrm{AB}$ & 139.12 & A & 141.34 & A \\
\hline VII & Ivermectin $(1350 \mu \mathrm{g})+$ Abamectin $(750 \mu \mathrm{g})$ & 167.84 & $\mathrm{ABC}$ & 135.96 & $\mathrm{AB}$ & 122.52 & A & 129.40 & A \\
\hline
\end{tabular}

Means values followed by the same letter on the same colun do not differ significantly at a $95 \%$ reliability level.

susceptible to intoxication, such as calves under four years old. They affirmed that dosages up to five times the recommended dose would not be lethal, even though severe signs of intoxication, and even deaths, occur when abamectin is used in dosages much greater (five to 10 times) than what is recommended or administered to animals presenting higher sensitivities. The results of the present study are similar to those obtained by the aforementioned authors, confirming their findings regarding safety of avermectins, even in susceptible animals.

However, it is essential to reinforce that these results do not allow for the recommendation of abamectin use in calves under 30 days old. For these young calves, new studies must be performed. Nonetheless, intoxication does not seem to occur in the majority of susceptible animals that received abamectin. It is important to note that other hypotheses must be considered in clinical cases suggestive of intoxication diagnosed in the field, similar to those reported by Seixas et al. (2006).

\subsection{Cerebrospinal fluid}

All samples collected, from both treated and control groups, were clear and colorless and within normal ranges as described by Kay et al. (1974), Wright (1978) and Bailey and Vernau (1997). For the remaining parameters evaluated in CSF samples, it is important to reinforce that no statistically significant differences $(p>0.05)$ were observed, across all experimental dates, in density, glucose, LDH, CK and total protein.

For $\mathrm{pH}$, it was possible to verify that values varied between 6.8 and 8.0, with significant changes observed before treatment (day zero) and on the first DPT for groups T02 (abamectin $200 \mu \mathrm{g} / \mathrm{kg}$ ) and T04 (abamectin $600 \mu \mathrm{g} / \mathrm{kg}$ ), as well as for group T05 (ivermectin $450 \mu \mathrm{g}$ / $\mathrm{kg}+$ abamectin $250 \mu \mathrm{g} / \mathrm{kg}$ ) and the remaining groups (Table 3 and Figs. 2a and b). Still, it is essential to highlight that $\mathrm{pH}$ results did not exceed normal physiological values given by Jean et al. (1997).

Concerning the cellular composition in CSF samples, a significant difference ( $\mathrm{p} \leq .05$ ) was observed only on 1 DPT for average leukocytes of animals that received ivermectin $(450 \mu \mathrm{g} / \mathrm{kg})+$ abamectin $(250 \mu \mathrm{g} /$ $\mathrm{kg}$ ) compared to the remaining groups (Table 3 and Figs. 2a and b). These CSF values can be considered normal and are similar to observations by Welles et al. (1994) and Jean et al. (1997).

Elevated average levels ( $\mathrm{p} \leq .05$ ) of CSF aspartate aminotransferase $(\mathrm{u} / \mathrm{L})$ were observed in calves from groups T02 (abamectin $200 \mu \mathrm{g} / \mathrm{kg}$ ) and T05 (ivermectin $450 \mu \mathrm{g} / \mathrm{kg}+$ abamectin $250 \mu \mathrm{g} / \mathrm{kg}$ ) in comparison to average levels observed in animals treated with abamectin (400 and $600 \mu \mathrm{g} / \mathrm{kg})$ and the ivermectin + abamectin $(900 \mu \mathrm{g} / \mathrm{kg}+500 \mu \mathrm{g} / \mathrm{kg}$, $1350 \mu \mathrm{g} / \mathrm{kg}+750 \mu \mathrm{g} / \mathrm{kg}$ ) association. According to Feldman (1989), Chrisman (1985), Osuna (1992) and Lutsar (1994), increases in the activity of the AST enzyme in this location can constitute an important indicator of active necrosis of the CNS of vertebrates.

However, despite the significant difference ( $\mathrm{p} \leq 0.05$ ) found between groups T02 (abamectin $200 \mu \mathrm{g} / \mathrm{kg}$ ) and T05 (ivermectin $450 \mu \mathrm{g}$ / $\mathrm{kg}+$ abamectin $250 \mu \mathrm{g} / \mathrm{kg}$ ) for AST, it is essential to reinforce that values quantified for the aforementioned groups did not differ significantly $(\mathrm{p}>0.05)$ regarding average levels of CSF aspartate aminotransferase $(\mathrm{u} / \mathrm{L})$ present in calves belonging to the control group (T01 - saline solution). Therefore, this result does not support the 

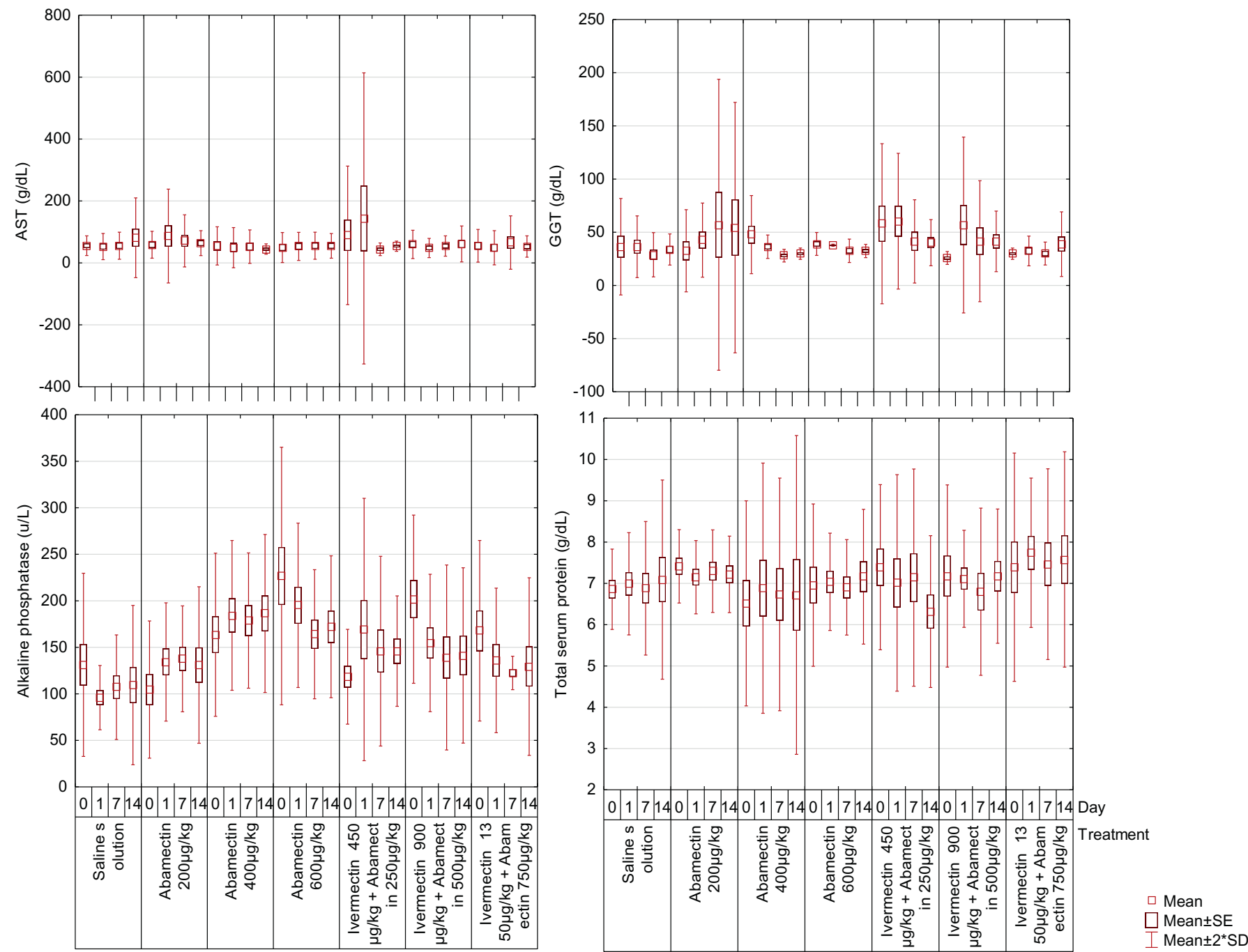

Fig. 3. Boxplot of the biochemical parameters of bovine animals under 30 days submitted to different concentrations of avermectin.

inference that increases observed in groups T02 and T05 occurred as a consequence of synthetic chemical treatments performed on these animals.

\subsection{Biochemical blood tests}

Total proteins analyzed stayed within normal limits for all experimental dates (Kaneko et al., 1997). Regarding AST, the majority of values were within normal ranges, while some were above normal. For GGT, all values were above those cited as normal for bovines (Kaneko et al., 1997). Nonetheless, it is possible to verify that no significant differences $(\mathrm{p}>0.05)$ occurred among biochemical values of total proteins, AST and GGT obtained from animals treated with different formulations containing abamectin and/or ivermectin compared to the control group across all observational dates (Table 4 and Fig. 3).

As for alkaline phosphatase, significantly greater $(\mathrm{p} \leq 0.05)$ average values were found for groups of calves treated with 400 and $600 \mu \mathrm{g} / \mathrm{kg}$ abamectin than in the other groups. However, it is essential to reinforce that significant differences ( $\mathrm{p} \leq 0.05$ ) between groups regarding mean alkaline phosphatase values were already observed before the start of treatments (Table 4 and Fig. 3). In addition, alkaline phosphatase values observed in experimental calves (treated and control) throughout the experiment remained within normal parameters for the target species (Kaneko et al., 1997), supporting the finding that reported significant differences had no clinical significance.

\section{Conclusion}

Even though it is well known that abamectin may lead to cases of intoxication in bovines less than four months old, based on results obtained in the present study, it was not possible to observe adverse effects, including neurological toxicity, biochemical and cerebrospinal fluid related, in calves $<30$ days old that had received up to $600 \mu \mathrm{g} / \mathrm{kg}$ of an abamectin-based formulation, as well as an association containing up to $1350 \mu \mathrm{g} / \mathrm{kg}$ and $750 \mu \mathrm{g} / \mathrm{kg}$ ivermectin + abamectin, respectively,. The level and activity of the multiple drug resistance gene (MDR1 - ABCB1) present in the calves may have influenced the results found in this study. Future studies should be carried out.

\section{References}

Albert, J., Lingle, D.H., Marder, E., O'Neil, M.B., 1986. A GABA-activated chloride condutance not blocked by picrotoxin on spiny lobster neuromuscular preparetions. Br. J. Pharmacol. 87, 771-779.

Bailey, C.S., Vernau, W., 1997. Cerebrospinal fluid. In: Kaneko, J.J., Harvey, J.W., Bruss, M.L. (Eds.), Clinical biochemistry of domestic animals. 27. Academic. Cap., San Diego, pp. 785-827.

Benz, G.W., Cox, J.L., 1989. In: Campbell, W.C. (Ed.), Use of abamectin in cattle. Ivermectin and Abamectin. Springer Verlag, New York, pp. 230-233.

Bissonnette, S., Paradis, M., Daneau, I., Silversides, D.W., 2008. The ABCB1-1Delta mutation is not responsible for subchronic neuro-toxicity seen in dogs of non-collie breeds following macrocyclic lactone treatment for generalized demodicosis. Vet. Dermatol. 20, 60-66.

Braund, K.G., 1994. Diagnostic techniques. In: Braund, K.G. (Ed.), Clínical syndromes in 
veterinary neurology. St Louis: Mosby, pp. 333-421.

Burg, R.W., Miller, B.M., Baker, E.E., Birnbaum, J., Currie, S.A., Hartman, R., Kong, Y.L., Monaghan, R.L., Olson, G., Putter, I., Tunac, J.B., Wallick, H., Stapley, E.O., Oiwa, R., Omura, S., 1979. Avermectins, new family of potent anthelmintic agents: producing organism and fermantation. Antimicrob. Agents Chemother. 15, 361-367.

Button, C., Barton, R., Honey, P., Rickford, R., 1988. Avermectin toxicity in calves and an evaluation of picrotoxin as an antidote. Aust. Vet. J. 65, 157-158.

Chrisman, C.L., 1985. Investigações auxiliares especiais. In: Chrisman, C.L. (Ed.), Neurologia dos pequenos animais, pp. 63-96 (São Paulo:Roca).

Courtney, C.H., Roberson, E.L., 1995. Chemotherapy of parasitic disease. Anti nematodal drugs. In: Adams, H.R. (Ed.), Veterinary Pharmacology and Therapeutics, 7th ed. Iowa State University Press, Ames, pp. 916-922.

Fecht, S., Wöhlke, A., Hamann, H., Distl, O., 2007. Analysis of the canine MDR1-1 mutation in the dog breed Elo. J. Vet. Med. 401-405.

Feitosa, F.L.F., 2008. Semiologia veterinária: A arte do diagnóstico, 2nd ed. Roca, São Paulo (735 pp).

Feldman, B.F., 1989. Cerebrospinal fluid. In: Kaneko, J.J. (Ed.), Clinical Biochemistry of Domestic Animals, 4 ed. Academis Press, San Diego, pp. 835-865.

Fromm, M.F., 2004. Importance of P-glycoprotein at blood-tissue barriers. Trends Pharmacol. Sci. 25, 423-429.

Gama, F., 2003. G V. Proteinograma e caracteres físico-químicos e citológicos do liquor de cães com cinomose. Jaboticabal 50p. (Dissertação (Mestrado) Faculdade de Ciências Agrárias e Veterinárias, Universidade Estadual Paulista).

Geyer, J., Janko, C., 2012. Treatment of MDR1 mutant dogs with macrocyclic latones. Curr. Pharm. Biotechonol. 13, 969-986.

Geyer, J., Döring, B., Godoy, J.R., Moritz, A., Petzinger, E., 2005. De-velopment of a PCRbased diagnostic test detecting a nt230(del4) MDR1 mutation in dogs: verification in a moxidectin-sensitive Australian shepherd. J. Vet. Pharmacol. Ther. 28, 95-99.

Jean, G.S.T., Jean, K., Yvorchuk, S., Anderson, D.E., Moore, W.E., 1997. Cerebrospinal Fluid Constituents Collected at the Atlanto-Occipital Site of Xylazine Hydrochloride Sedated, Healthy 8-week-old Holstein Calves. Can. J. Vet. Res. 61, 108-112.

Kaneko, J.J., Harvey, J.W., Bruss, M.L., 1997. Clinical Biochemistry of Domestic Animals. 27. Academic Cap, San Diego, pp. 785-827.

Kay, W.Y., Israel, E., Prata, R.G., 1974. Cerebrospinal fluid. Vet. Clin. N. Am. 04, 419-435.
Lankas, G.R., Gordon, L.R., 1989. Toxicology. In: Campbell, W.C. (Ed.), Ivermectin and Abamectin. Springer Verlag, New York, pp. 89-112.

Little, T.M., Hills, F.J., 1978. Agricultural experimentation designs and analysis. Wiley, New York 1978 (350p).

Lutsar, I., 1994. Enzymatic changes in the cerebrospinal fluid in patients with infections of the central nervous system. Acta Paediatr. 83, 1146-1150.

Marzolini, C., Paus, E., Buclin, T., Kim, R.B., 2004. Polymorphisms in human MDR1 (Pglycoprotein): recent advances and clinical rele-Vance. Clin. Pharmacol. Ther. 75, 13-33.

Mealey, K.L., 2004. Therapeutic implications of the MDR-1 gene. J. Vet. Pharmacol. Ther. $27,257-264$.

Mellin, T.N., Busch, R.D., Wang, C.C., 1983. Postsynaptic inhibition of invertebrate neuromuscular transmission by avermectin $B_{19}$. Neuropharmacology 22, 89-96.

Osuna, E., 1992. Efficacy of cerebrospinal fluid biochemistry in the diagnostic of brain insult. Forensic Sci. Int. 52, 193-198.

Prichard, R., Ménez, C., Lespine, A., 2012. Moxidectin and avermectins: consanguinity but not identity. Int. J. for Parasitology: Drugs and Drug Res. 2, 134-153.

Sas INSTITUTE, 1996. The SAS System for Windows: version 6.12 (compact disc). SAS. Institute, Cary. NC, USA.

Schinkel, A.H., Mayer, U., Wagenaar, E., Mol, C.A., Van Deemter, L., Smit, J.J., Van Der Valk, M.A., Voordouw, A.C., Spits, H., Van Tellingen, O., Zijlmans, J.M., Fibbe, W.E., Borst, P., 1997. Normal viability and altered pharmacokinetics in mice lacking mdr1type (drug-transporting) P-glycoproteins. Proc. Natl. Acad. Sci. 94, 4028-4033.

Seixas, J.N., Peixoto, P.V., Armién, A.G., Jabour, F.F., Brito, M.F., 2006. Aspectos clínicos e patogenéticos da intoxicação por abamectina em bezerros. Pesquisa Veterinária Brasileira. 26, 161-166.

Stober, M., 1993. Identificação, anamnese, regras básicas da técnica do exame clínico geral. In: Exame clínico dos bovinos, 3. ed. Guanabara Koogan, Rio de Janeiro (419 pp).

VICH, 2008. Guideline 43 - Target Animal for Veterinary Pharmaceutical Products Julho de.

Welles, E.G., Pugh, D.C., Wenzel, G.W., 1994. Composition of cerebrospinal fluid in healthy adult llamas. Am. J. Vet. Res. 55, 1075-1079.

Wright, J.A., 1978. Evaluation of cerebrospinal fuid in the dog. Vet. Rec. 103, 48-51. 